\title{
El paradigma educativo en la licuidad contemporánea
}

\author{
Seber Ugarte CAlleja \\ Universitat Abat Oliba-CEU, Barcelona \\ seber@psicrea.com
}

\begin{abstract}
Resumen:
Toda aproximación al sistema educativo contemporáneo resulta, en estos momentos, una aventura llena de incertidumbres. Hace no tantos años solíamos pensar que a cambio de paradigma se hacía necesario un cambio en las herramientas; sin embargo, el bloqueo al que la infoxicación y los argumentos externos nos tienen acostumbrados hacen inviable una línea de equilibrio entre la educación y lo que nos está aconteciendo.

Desde este ensayo hemos abordado la cuestión del criterio dentro de la educación y sobre todo, la necesidad de incorporarlo de forma creativa, activando nuestra capacidad de asimilación, nuestra inteligencia emocional y nuestra plena consciencia.
\end{abstract}

Palabras clave: Comunicación; Social Media; Educación líquida; Sistema educativo; Creatividad; Criterio; Inteligencia Emocional; Fluir; Csikszentmihalyi; Robinson; Goleman.

\section{The educational paradigm within modern liquidness}

\begin{abstract}
:
Approaching the modern educational system is currently a journey full of uncertainties. Not so many years ago, we used to think that a change of paradigm required a change of tools; however, blockade by 'infoxication' and outside arguments make it unfeasible to find a balance between education and what is happening to us.

This essay addresses the topic of judgement in education and, above all, the need to use it creatively, enabling our understanding capacity, our emotional intelligence and our full awareness.
\end{abstract}

Key Words: Communication; Social Media; Liquid Education; Education System; Creativity; Criterion; Emotional Intelligence; Flow; Csikszentmihalyi; Robinson; Goleman.

\section{Referencia normalizada:}

Ugaarte Calleja, S. (2014): El paradigma educativo en la licuidad contemporánea. Historia y Comunicación Social. Vol. 19. Núm. Especial Enero. Págs. 41-52

Sumario: 1. Introducción. 2. La (dis)función contemporánea. 3. Metodología. 3.1. Elementos y Licuidad Educational. 4. Propuesta in time.

\section{Introducción}

Se puede afirmar que la fluidez baumaniana cuestiona la fiabilidad de los recursos individuales frente a la globalidad niveladora que supone una tabla rasa tanto a nivel sociológico como cultural o económico. El agotamiento del mundo de Bauman, (2008:23), nos remite directamente a un peculiar encuentro con la pérdida de identi- 
dad, de corporalidad, a partir de una territorialidad en la que nuestra sociedad, como tal, parezca disolverse. Desde lo macro a lo micro la pregnancia interpersonal acontece desvanecida y fragmentada. Los límites de la escena global se están desmembrando al tiempo que producen una serie de acontecimientos en los que el individuo poco o nada tiene que hacer ante la situación que creada. El espacio absoluto, en el que nos encontramos, produce un abuso constante por parte de las instituciones frente al individuo, y conecta la inconsistencia del sistema actual de comunicación con la ficción utópica y evasiva de la falacia, a propósito de una promesa de libertad, del todo difusa.

Vivimos una realidad social, pero también, educativa caduca y sobresaturada en la que el tratamiento del alumno es entendido como la suma de una serie de habilidades comunes sin tener en cuenta la impronta que eso supone para cada uno de nosotros. Una respuesta sistemática en el ejercicio del saber que no ayuda a que cada individuo sea lo que en realidad siente que quiere ser.

Esta ecuación tiene en su formula a lo global y a la discutida individualidad-como sinónimo de especialización-y, como parece, está suponiendo una degradación de los modelos. Es decir, un cambio en la orientación de nuestros objetivos vitales, profesionales y culturales. La durabilidad y la constancia han dado paso a una pretenciosa intensidad efímera como forma de comprensión del mundo. El cambio del esfuerzo por la dinámica del llegar a conseguirlo, es algo que nos hace limitados en función de aquello que parece se pretende de nosotros como colectividad; un fin que deja elípticos los medios y cuyo resultado difumina la observación y pensamiento del recorrido.

Nos estamos convirtiendo en una colectividad más global pero también más abstracta. Un mercado en el que la soberanía del Estado está siendo subrogado a un continuo desplazamiento. Una multiplicidad de (des)gobiernos y de pretendidas identidades que sobre lo local desplazan el verdadero sentido del conjunto y de sus sinergias de solidaridad y pregnancia. La cuestión principal en las que nos introducimos en este análisis es, precisamente, la necesidad en la obtención de criterio real en el que apoyarse, teniendo en cuenta el desarrollo cognitivo y emocional del individuo, más a allá de los resultados cuantificables de los sistemas evaluativos contemporáneos.

Desde hace algunos años, venimos hablando de crear un sistema global, basado en unos criterios comunes de intercambio, en los que la reflexión pasa por hacer frente a una educación construida desde un sistema basado en la producción -capitalistadel conocimiento. Sin embargo, estamos percibiendo que situarnos fuera del artificio económico, sobre todo atendiendo al declive del modelo en crisis, nos está precipitando hacia un espacio lleno de carencias en cuanto a educación se refiere. El binomio formado por la sociedad del conocimiento y la tecnología, junto con los operativos comunicacionales contemporáneos, plantea una forma de entender la vida, de pensarla, que nada o poco tiene que ver con el paradigma que nos ha precedido; a no ser que la máxima fordiana esté desarrollándose en una producción desmesurada de los horizontes más allá de lo económico. 
En este contexto, el sistema de educación actual realiza comprometidos retos en los que las instituciones sumergen a los alumnos en un entramado de limitaciones, normativas y pretendidas innovaciones de extraño recorrido y escasa pedagogía. En comunicación, por ejemplo -área en la que desenvolvemos nuestra actividad docente-, los ámbitos desarrollados por los medios han desplegado un imaginario de pensamiento utópico, donde el individuo queda desprotegido, carente de fuerza y sobre él, se ejerce un profundo relativismo sistémico: Se puede afirmar, que el sistema acomoda a sus intereses económicos los valores éticos al disolver las obligaciones sociales, la solidaridad como compromiso y hasta la noción ética. La actuación de los sistemas educativos en esta nueva realidad resulta desalentador. El abandono institucional o la falta de imaginación hacen que estas actuaciones se instalen en una situación que no se terminan de comprender, asumiendo signos de ignorancia y retos diferentes a los exigidos para hacer frente al avance de una colectividad. La educación parece considerarse como un procedimiento menor en la construcción del propio tejido evolutivo y social. Analfabetos en muchos sentidos, sumidos como estamos en la retórica del ego, nos estamos replegado en una zona de confort sin precedentes en la que al parecer hemos dejado de lado los logros sociales, culturales y personales que nos han precedido.

Por otro lado, resulta contradictorio comprobar como la capacidad biológica de la cognición se desborda por un incesante flujo de información. Los recursos, en las sociedades avanzadas, son exponencialmente divergentes a las necesidades pero, sobre todo, a nuestra capacidad de asimilación limitada biológicamente hablando. La divergencia cognitiva, tecnológica y cultural se suma, en estos días, a la obtención sin pérdida de tiempo de rédito inmediato, ya que toda demora parece considerarse como un estigma. Consumo y producción sistemática que nos está sumiendo en una de las mayores crisis de identidad producto de un «éxito» que en ocasiones pone sobre la mesa el tema de nuestras limitaciones humanas. El conocimiento que somos capaces de generar es inversamente proporcional a nuestra capacidad para asimilarlo. Si es cierto que todas los cambios traen consigo una pérdida selectiva de lo asimilado, es necesario ser capaces de entender que el nuevo paradigma se está instalando sobre una base de incultura existencial preocupante y que la progresión es exponencialmente mayor a la que concurriera en cualquier otra época anterior. No está de más pensar que esa selección toca de lleno a nuestros sistemas educativos y lo hace donde más le duele a la cultura: creando analfabetos funcionales, capaces de resolver, sí, pero no de experimentar a través de un conocimiento y criterio propios lo asimilado. Aprender a aprender, reza como uno de los epígrafes en nuestro sistema actual universitario, sin embargo se hace necesario un: aprender a pensar. No solo como forma de comprender(nos) sino como espacio empático en la comprensión del otro y por lo tanto, del mundo que estamos construyendo.

Es necesario pensar en la educación como una creación en el tiempo, un procedimiento de asimilación progresivo, extendido sobre nuestra propia experiencia que requiere de paciencia, fracasos y, sobre todo, de adaptación y flexibilidad. Al hablar de educación debemos dejar de lado los resultados perentorios pues ellos anulan algo 
esencial para el conocimiento como es la capacidad humana de obtener y desarrollar criterio. Educar en la abundancia y en la necesidad desbordada de la obtención de resultados, es asumir que el conocimiento funciona como una competencia epidérmica que justifica la flotabilidad en superficie de las cosas.

En cierta medida el ser consciente de la situación nos lleva a plantearnos las siguientes cuestiones: primera, ¿Qué modelo de educación es posible con esta percepción social impaciente en la que culturalmente estamos avanzando a través de los espacios competitivos y la imperiosa necesidad en la obtención de resultados? Segunda, ¿Cuál sería el papel de la educación dentro de este marco social? Y en tercer lugar, ¿Puede ejercer de contrapeso la educación para equilibrar esta mentalidad imperante?

\subsection{La (dis)función contemporánea}

El valor del conocimiento se experimenta a través de su durabilidad, es decir, en su capacidad de ser transformado e incrementado en el tiempo. La formación de la persona culta es algo que se adquiere no solo a través de un esfuerzo constante, si no también a propósito de una progresión temporal y de una profunda reutilización de lo aprendido.

La extraña licuidad de lo contemporáneo nos enfrenta a un sistema en el que lo duradero no tiene atractivo y produce una sensación de brevedad existencial, causante de espacios de frustración en los que no solo no nos reconocemos, si no en los cuales estamos inmersos inconscientemente. El compromiso, las responsabilidad o la necesidad son supuestos de un estadio posesivo y acumulativo que no es entendido, en estos momentos, como valores en sí mismos. No se trata de poseer en cuanto que fisicidad. Más allá de la posesión no podemos entender la vida y mucho menos la educación, como un ameno y ligero discurso de sensaciones efímeras, ya que si lo hacemos corremos el peligro de asumir el aprendizaje como una mercancía más. Vencer el reemplazar lo conseguido por el hacer crecer, conviene más allá del esfuerzo que trae consigo.

En estos momentos, existe una figura errática en la que el conocimiento aparece como el resultado de un proyecto para olvidar más que como un valor determinarte en nuestra forma de entender y de aprehender el mundo. La volatilidad del ejercicio es incierta e incluso caótica y trabaja con un esquema de resultado cortoplacista que fija sus intereses en función de ser alcanzados en el menor tiempo posible. En este sentido, la velocidad es una importante herramienta y si bien se hace interesante en cuanto que movimiento, es indudable que la reflexión exige pararse para ver en perspectiva analizando los contenidos y los pasos dados. Nuestro presente no se puede convertir en un espacio de transiciones emocionales permanente en el que la falta de verdaderos estadios de la emoción nos lleve a determinar una soledad carente de toda lógica.

Posiblemente uno de los asuntos más perturbadores a los que nos enfrentamos sea la capacidad social para experimentar, a un ritmo vertiginoso, la adecuación interesada de estar siempre a la última. La formación en continuo estado de reciclaje se 
convierte así en una trampa, puesto que estar en permanente formación representa no desarrollar lo aprendido o por lo menos, aquello que es la base de nuestro saber. El nuevo enfoque desplaza, a favor de la inquietante evolución geométrica del conocimiento, todas sus energías.

Nuestro sistema educativo arrastra una serie de premisas ancladas en la Revolución Industrial. Con una separación por materias jerarquizadas, las asignaturas se desarrollan en función de una prioridad racional unidireccional, dentro de áreas poco permeables e indispuestas a la interrelación. Por ello, orientar nuestra cognición con criterio y sentido personal dentro de la cultura de masas o el conocimiento colectivo resulta cada vez más complicado y costoso. Es una especie de alienación circunstancial que pone en cuestión la capacidad del individuo para, en un futuro, tomar decisiones globales y democráticas pero sobre todo interrelacionadas.

La excesiva especialización no dejará de ser una fuente de conocimiento para la ciencia y la tecnología, incluso para la humanidades. Sin embargo, nuestro discurso intenta ir más allá del ámbito académico, centrándose en la educación como forma de acceso al mundo, es decir, a partir de una ética que contempla el discernimiento y el criterio de lo que significa estar formado como ciudadano. La predisposición a la privacidad, de esta nueva sociedad del conocimiento, tiende a reducir, la alternancia de ocio y consumo a estadios paralelos en torno al trabajo en cuanto a producción, al convertir nuestras dinámicas vitales en un bucle.

La ilusión de la nueva utopía en la que la «sociedad del conocimiento», tal y como la contextualizase Peter Drucker (1969), resulta interesante. Drucker partiendo de la «sociedad de la información» acuñada por Fritz Machlup (1962), partía de la base que el conocimiento es útil en tanto que no tiene un valor de cambio y plantea que el saber alberga una dinámica económica para el mercado llena de posibilidades. La productividad de ese saber es una limitación del propio conocimiento, pero no lo es para el contexto en el que se desarrolla la teoría económica tardo-capitalista y se podría decir, que ese conocimiento al que se refería Ducker, es hoy uno de los parámetros discursivos más trascendentes. Tras la afasia de los mercados, el colapso de las fuentes de energía, la producción low cost y la obsolescencia de las actividades tradicionales se puede interpretar que la «sociedad del conocimiento» es el único paradigma de lo posible -pensemos en otras alternativas contemporáneas-, y enseguida nos daremos cuenta que es casi imposible ir más allá de la utopía.

La sociedad del conocimiento junto al sistema, dan por sentado que el individuo asimila y entiende el mundo que le rodea, e interpreta que esa premisa es suficientemente sugerente como para provocar el hecho del conocimiento como tal. Sin embargo, debemos entender que el conocimiento no es -solamente-información si no una transcripción, comprensión y reflexión por parte de nuestro cerebro. Es la razón, la imaginación y su capacidad para discernir la que tanto la filosofía como la ciencia utilizan en la proceso que define al propio conocimiento, situando ambos espacios como herramientas conectadas. 
Pero ¿es cierto que nuestra sociedad se encamina hacia un conocimiento articulado por las tecnologías y su acceso? o por el contrario, ¿creemos en una fuga hacia delante sin plantearnos la realidad del saber disminuido en la que parece que nos encontramos? Antoni Brey (2011:62) en su ensayo: La sociedad del la ignorancia nos plantea esta idea ajustada al modelo actual, al decir: "La sociedad de la ignorancia es el corolario inevitable del mundo que hemos construido, o más bien que se ha ido formando a nuestro alrededor, porque aunque es obra de nuestras acciones no lo es de nuestras voluntades».

El desbordamiento de la información, al que nos tiene acostumbrados la sociedad contemporánea, no hace más que alterar nuestro discernimiento, abocándonos a una visión utópica, veloz y frustrante del saber. Esa desmotivación producida por el «exceso», es lo que Brey plantea como uno de los parámetros principales en lo que él denomina sociedad de la ignorancia. En segundo término, Brey, expone que la multitarea, entendida como la capacidad actual para responder al mismo tiempo a una ingente cantidad de recursos y herramientas, está creando una disfuncionalidad en el pensar propia de una desconcentración del propio proceso. A ello se le une la capacidad exponencial en la creación de una red de socialización permanente y casi siempre creciente. El entorno de red-artificial nos plantea una nueva patología del ego, puesto que nuestro impulso socializador está focalizando y abriendo nuestro espectro de relaciones de una manera igualmente exponencial. Nada queda establecido en la intimidad y nos estamos convirtiendo en entidades conectadas, virusadas en paralelo, que poco tienen que ver con la vivencia de la realidad y los aspectos empáticos que ésta conlleva. Sin ánimo de ser apocalípticos, la inmersión en el espacio virtual parece traer consigo una creciente intensidad por la auto-segregación capaz de inocular la confusión entre lo que es real y lo que acontece como espacio a propósito de esa virtualidad. Ello nos sitúa dentro de un territorio complicado donde si bien los expertos abundan, el saber se escapa de las regiones donde el conjunto y lo filosófico se retrotraen a sus propios círculos intelectuales. La mega-especialización contemporánea abandona, incluso entre los intelectuales, el estadio humanista para situarnos frente a un espacio de innovación permanente que demanda un tiempo progresivo, centrípeto y exponencial. Ni tan siquiera la universidad y el conocimiento crítico han escapado a esa doctrina en la que el castigo o el reconocimiento son activos propios del sistema. Y son en función de los círculos endogámicos afines a las financiaciones y los intereses dirigidos de empresas y centros educativos.

\section{Metodología}

La metodología empleada deriva de las preguntas planteadas al comienzo de este escrito. Al tratarse de un ensayo no hemos trabajado a partir de un operativo de campo basado en la observación sistemática de variables y la recogida de datos. La observación aunque directa está limitada a los diferentes trabajos que hemos realizados dentro de psicrea:research group, durante los dos últimos años, y que avalan la 
utilización de la pauta creativa, más allá de lo transversal, dentro de los programas educativos actuales.

La revisión de autores como M. Csikszentmihalyi, K. Robinson o D. Goleman, han sido por el momento la base utilizada para realizar nuestra propuesta sobre la implementación de los procesos creativos y las experiencias óptimas como áreas y herramientas claves en la obtención de una calidad educativa, basada en el discernimiento, la creación y el criterio.

\section{Propuesta in time}

En este escrito hemos pretendido escapar de esta inquietante especialización y de cierto autismo comunicacional imperante, abriendo las puertas a lo que en muchos casos se le ha negado una posibilidad constructiva. Si bien es cierto que cada día más proliferan los discursos sobre la creatividad como herramienta clave en los procesos de investigación, desarrollo e innovación, la experiencia recogida es bastante desalentadora. Nos estamos empeñando en instaurar una productividad a todas luces lastrada por unos intereses tardocapitalistas, sin ser conscientes que la emanación de la innovación, conforme a la experiencia creativa parten de la felicidad o fluidez de la misma, y sobre todo, a partir de un desarrollo de la inteligencia emocional que en muy pocos casos reconocemos.

El valor de la inteligencia emocional, a tenor de la creatividad, reside en propiciar fluidez [flow] a las «experiencias óptimas», en palabras de Csikszentmihalyi (2000: 21) «[...] es la manera en que la gente describe su estado mental cuando la conciencia está ordenada armoniosamente; gente que desea dedicarse a lo que hace por lo que le satisface en sìr; y éstas, a su vez, están interrelacionadas con el discurso que sobre la creatividad desarrolló Kevin Robinson (2009) en The Element. Ambos autores exponen y proponen una convergencia activa de los grados de asimilación del saber a través de experiencias que se asientan en una predisposición a la actuación activa del conocimiento basada en dicha creatividad.

En cierto sentido, la sociedad contemporánea ha generado un discurso escalofriante en el que la felicidad se construye sobre la represión de los deseos individuales. Es decir, estamos socializando(nos) en función de una dependencia controlada por poderes, sin preocuparnos en qué y cómo dirigimos nuestras propias libertades y sobre todo, nuestras formas de asimilación del mundo que nos rodea. Romper esas reglas es quedarse fuera y desvinculado del control de supervivencia tan importante para nuestras vidas. Lo que estamos produciendo -de forma irreal-, es una transformación del castigo por el -supuesto-placer, es decir, se nos induce a un ficción sobre nuestra calidad de vida, cuando lo que estamos experimentando es la frustración de nuestro propio sistema genético. Es importante entender que las pulsiones por si solas nos ahogan en su respuesta refleja y que lo instintivo debe establecerse también desde la cordura. Sin embargo, en pocas ocasiones pensamos que la creatividad es 
bastante cuerda en un sentido flexible de su terminología, puesto que establece, desde la libertad, un completo ordenamiento de la pulsión.

La creatividad es sensatez y «locura» en movimiento y por ello, es importante tenerla en cuenta para desarrollarla desde la niñez. La creatividad es una forma de conciencia capaz de ser colocada en un estadio diferente. Csikszentmihalyi (2000:63) hace la siguiente reflexión en este sentido:

Los componentes que necesitamos para entender cómo se puede controlar la conciencia creativa debemos enfocarlos en la atención. [...] la experiencia creativa depende de la manera en que utilizamos la energía psíquica (en la estructura de la atención), la cual, a su vez, está en relación con los objetivos y las intenciones. Estos procesos están conectados entre sí por la personalidad, o sea, por la dinámica representación mental que tenemos del sistema entero de nuestros objetivos. ${ }_{1}$

La personalidad está en relación con nuestros objetivos pero sobre todo con la analogía que los humanos establecemos con nuestra forma de aprender y de saber. Asumimos una exposición que nos mantiene en una entropía psíquica constante. Dicha formulación, desde nuestros primeros estadios educativos, nos refuerza la construcción de espacios para el miedo, el fracaso o la inquietud. Si no tenemos en cuenta estos parámetros estamos perdidos. El aprendizaje se basa precisamente en desplazar el miedo, asumiendo el fracaso como algo normal dentro de toda propuesta y actuar con la inquietud como argumento sólido en la búsqueda de saber, no como una pauta más de nuestras inseguridades.

El elemento principal capaz de construir experiencias óptimas dentro del aprendizaje es sin duda alguna darse cuenta que este tipo de experiencia tiene una finalidad en sí misma. Se trataría de una experiencia, en palabras de Csikszentmihalyi (2000:109), autotélica: «[...] actividad que se contiene en sí misma, que se realiza no por la esperanza de ningún beneficio futuro, sino simplemente porque hacerlo es en sí misma la recompensa». Es decir, la experiencia descrita actúa sobre la capacidad de motivar la propia creación sin centrarse en las consecuencias; al disfrutar de esa experiencia construimos, más allá del aprendizaje, una estructura de asimilación que fortalece la personalidad y activa el criterio.

La experiencia autotélica, o flujo, eleva el transcurso de la vida y del aprendizaje a otro nivel. La alienación da paso a la involucración, el disfrute reemplaza al aburrimiento, la impotencia da paso al sentimiento de control y la energía psíquica trabaja para reforzar la personalidad en lugar de perderse al servicio de unas metas extrínsecas. Cuando la experiencia es intrínsecamente gratificante la vida está justificada en su presente, en vez de ser el rehén de un hipotético beneficio futuro. ${ }_{2}$

Las experiencias óptimas se producen bajo una serie de actividades que, aunque nos parezcan anárquicas e incluso caóticas, poseen reglas que invitan al aprendizaje, determinan metas retroalimentándose. En este sentido, los juegos -la tan aludida gamificación $_{3}$ de la producción- nos ofrecerían oportunidades únicas para acometer los límites al construir experiencias novedosas, ya que la atención, si es enfocada sobre la actividad en sí misma no deja de mejorar la experiencia. Cualquier activi- 
dad capaz de transformar la realidad debe ser tenida muy en cuenta; la experiencia novedosa altera la consciencia y hace que ésta crezca. La creatividad conlleva precisamente esta forma de entender la conciencia, y el juego es definitivo en este sentido. El juego es creatividad definida en cuanto a que supone una capacidad de búsqueda dentro de la alteración (in)consciente, capaz de hacernos crecer frente a problemáticas planteadas.

A tenor de lo expuesto, la creatividad más que una habilidad en sí misma, es una actividad que debería poseer un alto rango de importancia dentro del aprendizaje. En estos tiempos en los que el emprendimiento parece ser la piedra filosofal sobre la que asentar nuestros problemas económicos, políticos y sociales, se hace perentorio ejercitarse de forma creativa. Más allá de su simple categoría transversal, la creatividad posee por sí misma un potencial inequívoco, al colocar bajo su radio de acción a todos los procesos de aprendizaje.

\subsection{Elementos y licuidad emocional}

La propuesta de Kevin Robinson (2009), de descatalogar nuestras enseñanzas eliminando las jerarquías entre asignaturas, sobre todo, poniendo en relación las diferentes áreas lógicas y plásticas nos parece acertada. La educación sería más empática, holística, flexible y abierta. En este paradigma las capacidades individuales tienen sentido más allá de las premisas que los mercados necesitan. Al difundir sentimientos positivos emergen por sí solos el rendimiento, el trabajo en equipo y la automotivación. Asuntos para los que la demanda contemporánea no sabe o no quiere encontrar solución en su globalidad.

Puede parecer simple -a primera vista lo es-, pero las habilidades emocionales traen consigo el eco de nuestros verdaderos intereses, de nuestro foco activo tanto sobre las emociones como en la propia producción creativa, laboral y social. Alejarnos de estos parámetros sería un error para nuestro sistema educativo pero también para el entramado social.

Nuestra propuesta es clara y simple. Al no existir ninguna tentativa de distancia debemos ser conscientes del poder de la creatividad como dispositivo de crecimiento. El efecto del análisis de los ecos y las autocríticas es una decantación que interrelacionan y hacen posible el encuentro de las disciplinas, de sus propias epistémicas con la propia actividad creativa y por ello no debemos de tener ningún reparo en experimentar en este sentido.

Como dijimos al inicio nos parece preocupante, la incoherente reversibilidad que los medios están teniendo en los menores. El gasto energético en la empleabilidad que los padres implementan en la creación de perfiles de sus bebés -en torno a un $81 \%$-, es un síntoma de cómo estamos orientando la visibilidad y la creatividad de nuestros hijos. No somos conscientes que las consecuencias de la sobreexposición en el imaginario de nuestros nativos digitales puede ser un inconveniente futuro. El

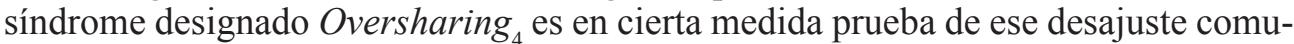
nicacional y licuado que nos acontece. Los datos del Informe Socialogue de IPSOS 
por ejemplo, sitúan a España como uno de los países con mayor penetrabilidad en la privacidad y los comentarios banales que sobre los demás generamos. Pensar en esta última idea con respecto de la educación es vital para comprender el tipo de sociedad que estamos construyendo en lo que respecta a la creatividad y a lo que hacemos visible para que ésta crezca.

\section{Conclusión}

Un cambio de ciclo como el actual exige un tratamiento diferente al que nos ha precedido históricamente. Desarrollarse en un espacio sobresaturado de información no es una tarea fácil, menos aún cuando ni tan siquiera somos capaces de percibir y de asimilar de esta información su forma global. El discurso contemporáneo nos está exigiendo un cambio en la manera de relacionarnos y entendemos que estamos evitando esa dirección. La poliédrica forma en la que estamos construyendo nuestra forma de relación -imágenes, narrativas, saberes, pensamientos y voces-, hacen fundamental una revisión, en perspectiva, no sólo de las disciplinas contenidas en el proceso educativo actual sino en todos los formatos de comunicación y producción de imaginario que nos acontece.

Es importante sopesar que mucho de lo que estamos escribiendo en estos momentos no es fruto más que de la transitoriedad de las prácticas y de las preguntas que nos estamos haciendo a tenor de los acontecimientos cotidianos. La disrupción siempre fue un elemento perturbador pero lo que resulta evidente es que los procesos casi siempre a priori son negativos, y nuestra propuesta es reorientar y sumar a esas herramientas comunicativas lenguajes y habilidades creativas con las que construir una mejor educación. Nos acontece una época de transición y cambio y debemos tener la posibilidad y la consciencias de que los argumentos simples son necesarios más allá de los burocráticos planes de estudio y normativas.

Si bien es posible pensar que el conocimiento actual se asienta en un territorio desbordado de informaciones y potencialidades; no deja de ser menos cierto, que la actuación desde una localización individual puede hacer que la comunidad genere la reorganización necesaria para que la estructura sea alterada. Socavar el sistema desde lo individual hacia lo colectivo, puede ser espejo de aquella forma originaria en la que el ser humano interactúa con lo colectivo,-empezando por la «revolución» en la esfera de lo personal-.

En empoderamiento de la colectividad es un recurso que debemos tener muy en cuenta en esta nueva sociedad del (des)conocimiento. De otro modo, sería imposible no percibir la amenaza que subyace a la pérdida de desarrollarnos como sujetos felices y realizados dentro de esa colectividad. En un sentido flexible la sociedad contemporánea magnifica la privacidad en detrimento de lo público, y no parece entender la banalización que ello conlleva para el sistema. La comunicación enmascarada por los medios es una forma de alienación en la que la presión del mercado 
-ocio, consumo- queda bajo los mismos parámetros de presión de la producción dentro de la especialización productiva y profesional. La obtención de criterio no puede ser un daño colateral de este enfoque puesto que la privatización de lo público permite minimizar las pretensiones sociales, culturales y por lo tanto educacionales, que delimitan nuestro desarrollo como individuos.

\section{Bibliografía}

CASTELLS, M. (1996-2003). The Information Age: Economy, Society, and Culture. Oxford: Blackwell Publishers.

CSIKSZENTMIHALYI, M. (2000). Fluir (Flow). Una psicología de la felicidad. Barcelona: Kairós.

DRUCKER, P. F. (1969). The Age of Discontinuity: Guidelines to Our Changing Society. Barcelona: Harper \& Row.

EDDWARS, R.; USHER, R. (2008). Globalisation \& Pedagogy, Place and Identity. New York: Routlegde.

GARDNER, H. (1993). Creative Minds. New York: Basic

GOLEMAN, D. (2001). La práctica de la inteligencia emocional. Kairós. Barcelona. HARGREAVES, A.; EARL, L.; RYAN, J. (1998). Una educación para el cambio. Barcelona: Octaedro.

MACHLUP, F. (1962). The Production and Distribution of Knowledge in the United States. Pricenton: Pricenton University Press.

MAYOS, G.; BREY, A. (eds.) (2011). La sociedad de la ignorancia. Barcelona: Península.

ROBINSON, K., (2009). The Element: How Finding Your Passion Changes Everything. New York: Penguin Group.

ZYGMUNT, B. (2007). Los retos de la educación en la modernidad líquida. Barcelona: Gedisa.

(2008). La sociedad sitiada. Buenos Aires: Fondo de Cultura Económica.

\section{Notas}

1. CSIKSZENTMIHALYI, M. (2000). Fluir (Flow). Una psicología de la felicidad. Barcelona: Kairós, p. 63. Traducido como: Finding Flow, es una compleja interrelación entre los desafíos y las habilidades. El fluir operativo se desarrolla en base, según en autor, a un equilibrio proporcionado entre dichos desafíos/habilidades. Sin embargo, en el libro no se tratan las debilidades obvias de tal simplificación.

2. CSIKSZENTMIHALYI (2000), Op. Cit., p. 112

3. Gamificación, a veces traducido al español como ludificación, es el uso del pensamiento y la mecánica de la jugabilidad en contextos ajenos a los juegos, con el fin de que las personas adopten cierto comportamiento. 
4. N. de T. Oversharing hace referencia al término anglosajón con el que se ha denominado este síndrome de compartirlo todo sobre tu hijo, tu gato, tu comida o tus plantas.

5. Informe Socialogue de IPSOS llevado a cabo en 24 países hace referencia a la creciente exposición a la privacidad y la construcción de imaginario en las que estamos inmersos las sociedades occidentales a través del social media.

\section{El autor}

Seber Ugarte Calleja, (Ph.D.) en Filosofía de la Universitat Autònoma de Barcelona. Adjunto al Vicerrector de Ordenación Académica en la Universitat Abat Oliba CEU y Dtor. del Dpto. de Comunicación y Profesor titular de Imagen, Creatividad y Dirección de Arte en la Universitat Abat Oliba CEU de Barcelona, (en excedencia). Profesor colaborados del Master en Psicocreatividad de la Universitat Autònoma de Barcelona. Investigador en el grupo psicrea:research UAO-CEU, Banco Santander. En paralelo a su actividad académica trabaja como fotógrafo y director de arte freelance especializado en obra plástica y arquitectura desde factordos estudio. 